\title{
COTOPOLOGY AND MINIMAL HAUSDORFF SPACES ${ }^{1}$
}

\author{
G. E. STRECKER AND G. VIGLINO
}

The concept of cotopology was introduced by J. de Groot, who showed that every regular space with a compact cospace is a Baire space and that for metrizable spaces, topological completeness can be defined in terms of compactness; specifically, topological completeness is equivalent to possession of a compact cospace (cf. [1] and [6]). The first result generalizes in a natural manner the two usual formulations of the Baire category theorem. Thus it is seen that a simply stated topological property of a cospace for some space can determine a more "sophisticated" property for the space itself.

In this paper it is shown that the property of having only Hausdorff cospaces is equivalent to Alexandroff's $H$-closed condition [2]. Using this, a simple characterization of minimal Hausdorff spaces is obtained, namely that a space is minimal Hausdorff if and only if it is identical with each of its Hausdorff cospaces. An application of these results yields a short proof of Banaschewski's result [3] that every semiregular Hausdorff space has a minimal Hausdorff extension.

Let $X=(X, \tau)$ be a topological space with $B[X]$ the collection of all open bases for $\tau$. Another topology $\tau^{\prime}$ defined on $X$ is said to be a cotopology of $(X, \tau)$ if there exists some $\beta \in B[X]$ such that the complements of closures of members of $\beta$ generate $\tau^{\prime}$, i.e. constitute an open subbase for $\tau^{\prime}$. The cotopology determined by the open base $\beta$ will be denoted by $\tau_{\beta}$, and the $\operatorname{cospace}\left(X, \tau_{\beta}\right)$ will be denoted by $X *(\beta)$. A cospace of $X$ which is homeomorphic to $X$ under the identity map is said to equal $X$. A space $X$ such that $X^{*}(\beta)=X$ for all $\beta$ $\in B[X]$ is called coinvariant.

1. H-closed, irreducible, and semiregular spaces. In the course of the paper the term (proper) extension of a topological space $X$ will denote a topological space which contains $X$ as a (proper) dense subspace. A Hausdorff space which has no proper Hausdorff extension is said to be $H$-closed (or absolutely closed) (cf. [2]). A topological space is said to be irreducible if every open set is connected, or, equivalently, if every nonempty open set is dense (cf. [7]). The complement in a topological space $X$ of the closure of a subset $A$ will be denoted by $E_{X} A$; thus $E_{X} A=X \backslash \mathrm{Cl}_{X} A$.

Received by the editors December 12, 1966.

${ }^{1}$ These results were independently obtained by the co-authors in their doctoral dissertations. 
LEMma 1.1. Every Hausdorff space which is not $H$-closed has an irreducible cospace.

Proof. Let $X$ be a Hausdorff space which is not $H$-closed. Then there exists some proper Hausdorff extension $Y$ of $X$. Let $p \in Y \backslash X$ and let $\left\{V_{\alpha}\right\}_{\alpha \in A}$ be the collection of $Y$-open neighborhoods of $p$. For each $\alpha \in A$, let $O_{\alpha}=E_{X}\left(V_{\alpha} \cap X\right)$. Since $Y$ is Hausdorff, $\theta=\left\{O_{\alpha}\right\}_{\alpha \in A}$ is an open cover of $X$. Refine $\mathcal{\theta}$ to an open base $\beta=\left\{B_{\delta}\right\}_{\delta \in D}$, i.e. each element of $\beta$ is contained in some element of $\theta$.

To show that $X^{*}(\beta)$ is irreducible it is sufficient to show that every pair of basic nonempty open sets has a nonempty intersection. We may assume that any such pair consists of $E_{X} \bigcup_{i=1}^{n} B_{\delta_{i}}$ and $E_{X} \cup_{i=1}^{m} B_{\delta_{i}^{\prime}}, B_{\delta_{i}} \in \beta, 1 \leqq i \leqq n ; B_{\delta_{i}^{\prime}} \in \beta, 1 \leqq i \leqq m ;$ since sets of this form constitute an open base for $X^{*}(\beta)$. Let $O_{\alpha_{i}} \in \mathcal{O}$ be such that $B_{\delta_{i}} \subset O_{\alpha_{i}}$, $1 \leqq i \leqq n$, and $O_{\alpha_{i}^{\prime}} \in \mathcal{O}$ be such that $B_{\delta_{i}^{\prime}} \subset O_{\alpha_{i}^{\prime}}, 1 \leqq i \leqq m$. Then

$$
\begin{gathered}
E_{X} \bigcup_{i=1}^{n} B_{\delta_{i}} \cap E_{X} \bigcup_{i=1}^{m} B_{\delta_{i}^{\prime}} \supset E_{X} \bigcup_{i=1}^{n} O_{\alpha_{i}} \cap E_{X} \bigcup_{i=1}^{m} O_{\alpha_{i}^{\prime}} \\
\supset \bigcap_{i=1}^{n} V_{\alpha_{i}} \cap \bigcap_{i=1}^{m} V_{\alpha_{i}^{\prime}} \cap X .
\end{gathered}
$$

But since $X$ is dense in $Y$, and each $V_{\alpha}$ is open, $\bigcap_{i=1}^{n} V_{\alpha_{i}} \cap \bigcap_{i=1}^{m} V_{\alpha i}^{\prime} \cap X$ is not empty.

Theorem 1.1. A Hausdorff space $X$ is $H$-closed if and only if $X^{*}(\beta)$ is Hausdorff for every $\beta \in B[X]$.

Proof. The sufficiency is immediate from the above lemma since it is clear that no irreducible space can be Hausdorff.

If $X$ is finite the necessity is clear. To establish the necessity otherwise, suppose that $X$ is $H$-closed and that for some $\beta \in ®[X], X^{*}(\beta)$ is not Hausdorff. Then there exist distinct points $p, q \in X$ such that every $X^{*}(\beta)$-neighborhood of $p$ has a nonempty intersection with every $X^{*}(\beta)$-neighborhood of $q$.

Let $X^{\prime}=X \backslash\{p, q\}$. Since $X$ is Hausdorff, for every $x \in X^{\prime}$ there exist $B_{x}, B_{p, x}, B_{q, x} \in \beta$ such that $x \in B_{x}, p \in B_{p, x} \cap E_{X}\left(B_{q, x} \cup B_{x}\right)$, and $q \in B_{q, x} \cap E_{X}\left(B_{p, x} \cup B_{x}\right)$. Thus, by hypothesis, for every $x \in X^{\prime}$,

$$
W_{x}=E_{X}\left(B_{q, x} \cup B_{x}\right) \cap E_{X}\left(B_{p, x} \cup B_{x}\right)
$$

is not empty.

Now it is possible to extend the space $X$ to the space $X \cup\{z\}$, where an open neighborhood base for the new point $z$ is $\left\{\{z\} \cup W_{x} \mid x \in X^{\prime}\right\}$. Since no $W_{x}$ is empty, $X$ is dense in $X \cup\{z\}$. 
Also if $x \in X^{\prime}, B_{x} \cap\left(\{z\} \cup W_{x}\right)$ is empty and $\left(B_{p, x} \cup B_{q, x}\right) \cap\left(\{z\} \cup W_{x}\right)$ is empty. Therefore $z$ has Hausdorff separation with every member of $X$ so that $X \cup\{z\}$ is a proper Hausdorff extension of $X$, which is impossible.

An open set $O$ in a topological space $X$ is regular open if $O=E_{X} E_{X} O$. A topological space which possesses a base of regular open sets is said to be semiregular.

Theorem 1.2. For a topological space $X=(X, \tau)$ the following are equivalent:

(i) $X$ is semiregular.

(ii) $X^{*}(\tau)=X$.

(iii) $X^{*}(\beta)=X$ for some $\beta \in B[X]$.

Proof. (i) $\Rightarrow$ (ii). Assume $X$ is semiregular. Let $\beta \in \mathbb{B}[X]$ be a base consisting of regular open sets. Then if $B \in \beta, E_{X} B \in \tau$ so that $E_{X} E_{X} B$ $=B \in X^{*}(\tau)$. Hence every open set in $X$ is open in $X^{*}(\tau)$.

(ii) $\Rightarrow$ (iii). Clear.

(iii) $\Rightarrow$ (i). Suppose that for some $\beta \in \mathbb{B}[X], X^{*}(\beta)=X$. Then $\left\{E_{X} O \mid O \in \beta\right\}$ is closed under finite intersections, so it constitutes a base for $X^{*}(\beta)$ and, consequently, for $X$. But each $E_{X} O$ is regular open since for any open set $A, E_{X} E_{X} E_{X} A=E_{X} A$. Hence $X$ is semiregular.

Lemma 1.2. In a topological space $X=(X, \tau)$, the closure in $X^{*}(\tau)$ of an open set in $X$ equals the closure of the set in $X$.

Proof. Let $O \in \tau$. The set $E_{X} O$ is open in $X^{*}(\tau)$ and disjoint from $O$. Hence $\mathrm{Cl}_{X^{*}(\tau)} O \subset \mathrm{Cl}_{X} O$. The inverse inclusion follows immediately from the fact that the topology of $X^{*}(\tau)$ is weaker than that of $X$.

Theorem 1.3. For any topological space $X=(X, \tau), X^{*}(\tau)$ is semiregular.

Proof. Denote $X^{*}(\tau)$ by $Y$. Let $O \in \tau$. Since $E_{X} O \in \tau, E_{X} E_{X} O$ is open in $Y$. Hence $E_{Y} E_{X} E_{X} O$ is open in $Y^{*}\left(\tau^{\prime}\right)$, where $\tau^{\prime}$ is the collection of sets open in $Y$. By the above lemma, $E_{Y} E_{X} E_{X} O=E_{X} E_{X} E_{X} O$ $=E_{X} O$. Thus each member of $\left\{E_{X} O \mid O \in \tau\right\}$ is open in $Y^{*}\left(\tau^{\prime}\right)$. But since $\left\{E_{X} O \mid O \in \tau\right\}$ constitutes an open base for $Y, Y^{*}\left(\tau^{\prime}\right)=Y$. Hence, by Theorem 1.2, $Y$ is semiregular.

2. Minimal Hausdorff spaces. A topological space $X=(X, \tau)$ satisfying a topological property $P$ is minimal- $P$ if there exists no topology on $X$ strictly weaker than $\tau$ satisfying property $P$ (cf. [4]).

Using the results of the previous section and a characterization of minimal Hausdorff spaces due to Katětov [8], namely that a space is 
minimal Hausdorff if and only if it is $H$-closed and semiregular, it is easy to deduce that a necessary and sufficient condition for a Hausdorff space to be minimal Hausdorff is that it be coinvariant. For, if $X$ is minimal Hausdorff it is $H$-closed, so that by Theorem 1.1, every cospace of $X$ is Hausdorff and hence must equal $X$ by the minimality of $X$. Conversely, if $X$ is coinvariant it is, by Theorems 1.1 and 1.2, both $H$-closed and semiregular and therefore minimal Hausdorff.

The following theorem shows that the property that every Hausdorff cospace equals the space is also equivalent to the minimal Hausdorff property. This result extends to other minimal spaces. Viglino has shown that a $P$-space, $X$, is minimal- $P$ if and only if every $P$-cospace of $X$ equals $X$ for the following topological properties, $P$ : (1) Hausdorff, (2) Urysohn, (3) regular, (4) completely regular, (5) normal, (6) completely normal, (7) locally compact (where minimal- $P$ is equivalent to compactness for properties (4) through (7)) [10].

Theorem 2.1. A Hausdorff space $X$ is minimal Hausdorff if and only if every Hausdorff cospace of $X$ equals $X$.

Proof. We need only show sufficiency. To do this we use the following characterization of minimal Hausdorff spaces which appears in Bourbaki [5]: A space is minimal Hausdorff if and only if every open filter base with a unique adherent point converges.

Suppose $X$ is not minimal Hausdorff. Then there exists an open filter base, $\mathcal{u}=\left\{U_{\alpha}\right\}_{\alpha \in A}$, with $\bigcap_{\alpha \in A} \mathrm{Cl}_{X} U_{\alpha}=\{p\}$; and an open neighborhood, $N_{p}$, of $p$ which contains no $U_{\alpha}, \alpha \in A$. Let $\beta=\mathscr{N} \cup \mathscr{T}$ where $\mathscr{N}=\left\{N \mid N\right.$ is open and $\left.p \in \mathrm{Cl}_{X} N\right\}$ and $\mathscr{T}=\{M \mid M$ is open and $M \subset E_{X} U_{\alpha}$ for some $\left.\alpha \in A\right\}$. Clearly $\beta \in B[X]$. We show that $X^{*}(\beta)$ is Hausdorff and not equal to $X$.

Let $p_{1}, p_{2}$ be distinct points of $X$ with $p_{2} \neq p$. Let $M \in \mathscr{T}$ be a neighborhood of $p_{2}$, whose closure contains neither $p_{1}$ nor $p$. Then $E_{X} M \in \mathfrak{N}$ so that $E_{X} E_{X} M$, as well as $E_{X} M$, is open in $X^{*}(\beta)$. Moreover, $p_{1} \in E_{X} M, p_{2} \in E_{X} E_{X} M$, and $E_{X} M \cap E_{X} E_{X} M=\varnothing$. Hence, $X^{*}(\beta)$ is Hausdorff.

We show $X^{*}(\beta) \neq X$ by showing that no $X^{*}(\beta)$-open neighborhood of $p$ is contained in $N_{p}$. It is sufficient to do this for sets of the form $\bigcap_{i=1}^{n} E_{X} B_{\alpha_{i}}, B_{\alpha_{i}} \in \beta=\mathfrak{N} \cup \mathscr{M}, 1 \leqq i \leqq n$, since they constitute an open base for $X^{*}(\beta)$. But if $\bigcap_{i=1}^{n} E_{X} B_{\alpha_{i}}$ is to contain $p$, each $B_{\alpha_{i}}, 1 \leqq i \leqq n$, must be an element of $\mathfrak{T}$. Hence, for each $i, 1 \leqq i \leqq n$, there exists a $U_{\alpha_{i}} \in \mathcal{U}$ such that $B_{\alpha_{i}} \subset E_{X} U_{\alpha_{i}}$. Thus $\bigcap_{i=1}^{n} E_{X} B_{\alpha_{i}} \supset \bigcap_{i=1}^{n} E_{X} E_{X} U_{\alpha_{i}}$ $\supset \bigcap_{i=1}^{n} U_{\alpha_{i}}$. Since $\mathcal{u}$ is a filter base, $\bigcap_{i=1}^{n} U_{\alpha_{i}}$ contains some member of $\mathcal{u}$. Hence since $N_{p}$ contains no member of $\mathcal{U}, N_{p}$ cannot contain $\bigcap_{i=1}^{n} E_{X} B_{\alpha_{i}}$. 
Theorem 2.2. A Hausdorff space $X=(X, \tau)$ is H-closed if and only if $X^{*}(\tau)$ is minimal Hausdorff.

Proof. Let $X$ be $H$-closed. Since the closure of a set in $X^{*}(\beta)$ contains the closure of the set in $X$ for all $\beta \in B[X], X^{*}(\beta)$ is $H$-closed for all $\beta \in B[X]$. In particular $X^{*}(\tau)$ is $H$-closed. Moreover, by Theorem $1.3, X^{*}(\tau)$ is semiregular. Hence $X^{*}(\tau)$ is minimal Hausdorff.

Conversely, suppose that $X^{*}(\tau)$ is minimal Hausdorff. Then it is $H$-closed, so that it has no proper Hausdorff extension. But if $\left(Y, \tau^{\prime}\right)$ is a proper Hausdorff extension of $X$, then $Y^{*}\left(\tau^{\prime}\right)$ is a proper Hausdorff extension of $X^{*}(\tau)$ since $\left(E_{Y} O\right) \cap X=E_{X}(O \cap X)$ for all $O \in \tau^{\prime}$. Therefore $X$ has no proper Hausdorff extension, i.e. $X$ is $H$-closed.

3. Minimal Hausdorff extension of semiregular spaces. Banaschewski [3] has shown that a Hausdorff space can be embedded densely in a minimal Hausdorff space if and only if it is semiregular. He constructed, for an arbitrary semiregular Hausdorff space, a minimal Hausdorff extension, based on the $H$-closed extension constructed by Katětov [9]. In Theorem 3.1, a method is given for obtaining a minimal Hausdorff extension of a semiregular space, $X$, given any $H$-closed extension of $X$.

Strecker has shown in his dissertation that every Hausdorff space can be embedded as a closed subset of a minimal Hausdorff space with the same weight and that every Hausdorff space has an $H$-closed extension with the same weight.

Lemma 3.1. Let $X=(X, \tau)$ be semiregular, and $Y=\left(Y, \tau^{\prime}\right)$ be any extension of $X$. Then $Y^{*}\left(\tau^{\prime}\right)$ is a semiregular extension of $X$.

Proof. By Theorem 1.3, $Y^{*}\left(\tau^{\prime}\right)$ is semiregular. Since $\left(E_{Y} O\right) \cap X$ $=E_{X}(O \cap X)$ for all $O \in \tau^{\prime}, Y^{*}\left(\tau^{\prime}\right)$ is an extension of $X^{*}(\tau)$. But since $X$ is semiregular we have, by Theorem 1.2 that $X=X^{*}(\tau)$.

Theorem 3.1. Let $X$ be semiregular, and $Y=(Y, \tau)$ be any $H$-closed extension of $X$. Then $Y^{*}(\tau)$ is a minimal Hausdorff extension of $X$.

Proof. By Theorem 2.2, $Y^{*}(\tau)$ is minimal Hausdorff and by the above lemma it is an extension of $X$.

\section{REFERENCES}

1. J. Aarts and J. de Groot et al., Colloquium co-topologie, Math. Centrum Amsterdam, Afd. Zuivere Wisk., 1964.

2. P. Alexandroff and P. Urysohn, Mémoire sur les espaces topologiques compacts, Verh. Nederl. Akad. Wetensch. 14 (1929), 1-96.

3. B. Banaschewski, Über Hausdorffsch-minimale Erweiterung von Räumen, Arch. Math. 12 (1961), 355-365. 
4. M. P. Berri, Minimal topological spaces, Trans. Amer. Math. Soc. 108 (1963), 97-105.

5. N. Bourbaki, Topologie générale, Hermann, Paris, 1951, pp. 858-1142.

6. J. de Groot, Subcompactness and the Baire category theorem, Nederl. Akad. Wetensch. Indag. Math. 25 (1963), 761-767.

7. A. Grothendieck and J. Dieudonné, Elements of algebraic geometry. I, Inst. Hautes Etudes Sci. Publ. Math. No. 4, 1960.

8. M. Katětov, Über H-abgeschlossene und bikompakte Räume, Casopis Pěst. Mat. Fys. 69 (1940), 36-49.

9. - On H-closed extensions of topological spaces, Časopis Pěst. Mat. Fys. 72 (1947), 17-32.

10. G. Viglino, A co-topological application to minimal spaces, Pacific J. Math. 27 (1968), 197-200.

TUlane University, University of Florida, Washington University AND WESLEYAN UNIVERSITY 